\title{
Design of a Low-power Smart Antenna System Algorithm
}

\author{
Aaron Don M. Africa, Raine Mattheus C. Manuel, Joshua Vincent G. Ligayo, Francis Xavier Asuncion, \\ Janos Lance Tiberio, Raymund Miguel Francisco A. Munchua \\ Department of Electronics and Communications Engineering \\ De La Salle University, Manila \\ 2401 Taft Ave., Malate, Manila 1004, \\ Philippines, aaron.africa@dlsu.edu.ph
}

\begin{abstract}
Advanced wireless communications technology available today requires smart antennas. The design of the antenna is the most important aspect to make a feasible signal transmission. Parameters of the antenna depend on the design of the antenna. This study tackles the idea of a smart antenna system model with low-power consumption. This study proposes a model for a low-power smart antenna. The presented model includes components such as microcontroller to achieve a low power model. The paper presents the simulation and model design of this system.
\end{abstract}

Key words: smart antenna, microcontroller, antenna array, radiation.

\section{INTRODUCTION}

Antenna theory is one of the fundamental principles in communication system. It involves designing and analyzing different types of antenna. Antenna is a metallic device that is cable to receive a transmission of signal. It can transmit and receive radio waves. In other words, antennas basically guide transmission signals. There are types of antennas, the receiving and transmitting antenna [1]. Antennas are widely used in communications. Usually, it is used in electronic communications and telecommunications applications. Modern antenna would not exist without the discovery of Maxwell's equations and the concept of electromagnetic waves. Electromagnetic waves enable the use of wireless communication which it consists of electric field and magnetic field [2].

Antenna arrays are the ways to provide efficient signals that is capable to detect it on different direction. Since single antennas is limited to the amount of bandwidth and directivity, arrays can be modified by manipulating its amplitude and phase distribution. These are called the weights of the array. When the antenna outputs are processed, signals are summed to the beam pattern of the antenna array $[3,4]$.

Nowadays, antenna designers are implementing an advancement of wireless communications design. Microcontrollers are one of the components that can advance the design of wireless communications. It can perform low power which it is efficient to low power devices that can withstand wireless communication systems [5].

\section{BACKGROUND OF THE STUDY}

With today's technology, antenna design has seen a rapid advancement in being able to transmit and receive a high-bandwidth of information. Researches often focus on increasing the rate of transfer of information usually, by increasing power. However, an approach to maximizing the transmission of signals may lie in the positioning of an antenna. An antenna has a beam pattern which determines a transducers sensitivity in different directions. The beam pattern of an antenna has a main lobe and side lobes. Usually, the main lobe is perpendicular to the transducer. The signal is at maximum at the main lobe whereas, side lobes contain very minimal signal and is often useless for demodulation [6]. Since antennas are stationary devices, often times the signal being transmitted from the transmitter to the receiver is not at maximum because the transmitter and receiver are not directly facing each other. This means that power is not being efficiently used and is being wasted in trying to transmit information from transmitter to receiver. However, a study on a self-reconfigurable antenna implementation may be a solution to this problem. A self-reconfigurable antenna implementation allows the antenna to steer its beam pattern to a desired direction [7]. The design of said antenna may change the direction of its beam pattern either through, signals be sensed or through human configuration. A key concept in the implementation of the smart antenna is through the radio electromagnetic spectrum broadband, in which there are the multiple transmitting antennas and the multiple receiving antennas [8]. Multiple antenna design implementation can help identify where the signal being transmitted is at maximum. Another key concept may lie in the improving the parallelism of transmissions [9]. This may be implemented via a rotating mechanism to ensure power efficiency in signal transmission. Another approach in the implementation of a smart antenna is through Mobile Ad Hoc Networks (MANET). MANET are autonomous self-configuring networks [10]. This may help in reconfiguring directional antennas to improve power efficiency of these systems. 


\section{STATEMENT OF THE PROBLEM}

An overlooked concept in antenna design implementation, is the beam pattern of antennas. Since antennas are stationary, the direction of the main lobe of the radiation pattern of a transmitter antenna is commonly not directly facing towards a receiving antenna, power is not being maximized. A common limitation of antenna design system is the power it uses. When a signal is being transmitted through a great distance, more power is needed. Instead of trying to provide antenna design systems with more power, a study can be made to a lot the resources in trying to direct the main lobe of the beam pattern to the receiving antenna. Not only will this system consume less power, in theory, it will also be less susceptible to noise. This study aims to design a smart antenna that will change its direction depending on the strength of the signal. The study aims to do this by providing the system with sensors that will detect the direction wherein the signal is at maximum and will shift its direction to face that signal.

\section{SIGNIFICANCE OF THE STUDY}

Power efficiency is a goal for most electronic circuits. An antenna is usually stationary, and the beam pattern does not change direction which means that a transmitting antenna will require more power the further a receiving antenna, not facing the transmitter antenna, is. In a smart antenna system, the antenna can detect where the signal is coming from or where the signal is strongest so that it can face that direction and lessen the power consumption of the system. The study will also contribute to understanding the beam pattern of an antenna and how to maximize power usage given the smart antenna system design. The system can make use of the direction of the main lobe of the antenna and use it to shift its direction. Another benefit of the smart antenna system design is less susceptibility to noise. If a receiving antenna is directly facing a transmitting antenna, there is less interference during the transmission and the signal will be less noisy. A signal with minimal noise is easier to demodulate and will need less power. A smart antenna system will lessen the interference and noise acted upon the signal being transmitted. Signals can be sent more clearly from the transmitting antenna to the receiving antenna.

\section{OBJECTIVES OF THE STUDY}

\subsection{General Objective}

To Design an Algorithm for a Low-power Smart Antenna System.

\subsection{Specific Objectives}

1) To utilize a MATLAB Simulation model of Antenna Signals

2) To make the Antenna System Power Efficient

3) To make the Antenna System Susceptible to Noise

4) To compare the power saved of the directional antenna compared to the lost due to motor control

\section{DESCRIPTION OF THE SYSTEM}

The Smart Antenna system design will be a power efficient system and will have lower susceptibility to noise. This will be achieved through the rotating mechanism and sensors from the receiving antenna and by using a high gain antenna configuration. The receiving antenna will detect where the desired signal is being projected the strongest and block other signals in order to receive the signal with the least noise possible. The Smart antenna system design will use a microcontroller and different sensors in order to determine the strongest desired signal. The Antenna Rotation will ensure that the clearest signal is received and transmitted. An RF Sniffer is a device that is used to detect the desired signal depending on the configuration and what signals are required. The Antenna system that will be developed in this research will make the RF Sniffer efficient by making it consume only less power. The system will be power efficient because the Antenna that will be designed is rotatable. Meaning it can obtain better gain easily. An application of the proposed system is to increase the efficiency in terms of gain and power of RF Sniffers in detecting signals.

\section{METHODOLOGY}

The system simulation will be implemented using a rotatable antenna head, controlled by a servo connected to a microcontroller outfitted with signal strength sensors. The signal strength sensors will monitor the surrounding environment's frequency spectrum using an RF sniffer which can detect frequencies from $433 \mathrm{Mhz}$ to $1000 \mathrm{Mhz}$ [11]. The output of this module will be fed to a Comparator block to perform the comparative analysis between regions and create a digital output to be fed to the microcontroller. This can be implemented using an arduino and the RF switch library through simulation [12]. The digital output will then be analyzed and fed to the rotational mechanism composed of a servo and a yagi-uda antenna due to its high directional properties [13]. A Matlab simulation of an antenna transmitter receiver tuned at $433 \mathrm{Mhz}$ will be created to scope the theoretical limit of the system [14]. The Yagu Uda antenna array will be tested by creating a transmitter at varying distances transmitting a random number generated binary pulse modulated at a $433 \mathrm{Mhz}$ to $1000 \mathrm{Mhz}$ carrier frequency shifted at different angles[15,16]. This test will allow the observation of the effect of varying the position of the transmitted signal on the rotating mechanism of the receiver[17]. The received signal will then be compared to a traditional stationary antenna subjected to the same conditions[18]. Figure 1 shows the block diagram of the system. 


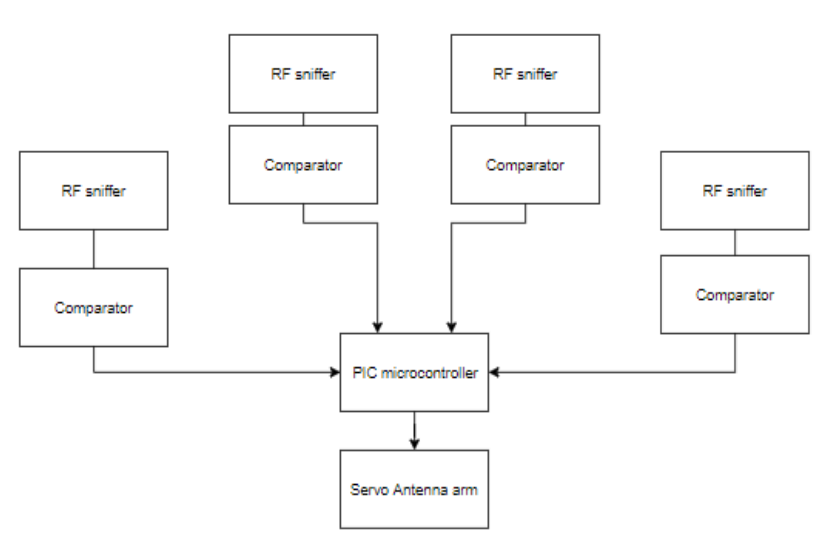

Figure 1: Block Diagram of the System

The system shown above consists of the RF sniffer modules that scan the environment for signals and their output is fed to a comparator circuit for analog to digital conversion. The data is then fed to the microcontroller for analysis, and the antenna is rotated accordingly. This system allows for maximum power reception that is adaptable with varying RF spectrum environments. This will also ensure the maximum transmitted power is received for efficient wireless transfer.

\section{REVIEW OF RELATED LITERATURE}

Antenna Array with Wide Angle Scanning Properties is a research paper by Noordin, Haridas, El-Rayis, Erdogan, and Arslan [19] that aimed to develop an antenna array with wide scanning properties. They focused on targeting portable devices and was able to create a 3-faced antenna linear array.Their antenna structure was simulated using CST Design Suite where results show that the main beam of the 3-faced antenna they made had a wide range of +- 70 degrees above the vertical position of the antenna. The problem with the results showed that the antenna must have wider beam width and that the side lobe levels increased which contributes the interference.

A research paper by Mani and Bose entitled Smart Antenna Design For Beamforming of UWB Signals in Gaussian Noise studied the problem of Ultra-Wideband (UWB) impulse beamforming when using linear array for the antenna. The problems were observed when the antenna is receiving multiple signals from different directions. They were able to create a smart antenna that is able to suppress interference by pointing the antenna's main beam towards the prestreed direction of the array and that it suppresses other signals coming from the antenna's other lobes. They were able to achieve this by UWB beamforming [20].

In a letter written by Celik and Iskander entitled Genetic-Algorithm-Based Antenna Array Design for a 60-GHz Hybrid Smart Antenna System, they were able to write an algorithm that allows a smart antenna to optimally adjust the beam pattern. This however, overlaps the array elements of the antenna that allows minimization of output power fluctuations. Because of this, they researched on how many optimal number of elements for an array that can provide minimal power fluctuation given a range. In this research, they also discovered the tradeoffs between acceptable level of power fluctuation and total received power [21].

Compact Smart Antenna System for Improving Probability of Detection is a paper that focused on developing a smart antenna system that would help reduce interference by pointing the radiation nulls towards interfering signals. The authors were also able to create a detection system by improving the probability of detection of an incoming signal, which helps the antenna's main lobe point towards the right direction [22].

In a thesis by Erik Öström entitled Building and experimentally evaluating a smart antenna for low power wireless communications [23], he was able to design a portable low-powered smart antenna that he calls SPIDA smart antenna, which is antenna shaped like a spider. The antenna was compared with an on-board PCB wire antenna, chip antenna, rod antenna, and a PCB antenna. Results showed that the antenna that he designed was cost efficient and small in size. The antenna uses a microcontroller and different sensors using common SMA antenna connectors and that the antenna can be controlled using the drivers that he developed programmed to the microcontroller. Comparing results with other types of antenna, the SPIDA antenna developed vastly outperformed the on-board micro-strip antenna.

New Reconfigurable Power Divider Based on Radial Waveguide and Cylindrical Electromagnetic Band Gap Structure for Low Power and Low-Cost Smart Antenna Systems is a paper by Boutayeb, Watson, and Kemp [24] where a design for a low power and low-cost smart antenna was proposed. The antenna has a reconfigurable radial waveguide that is excited by a central probe and excited with multiple feeding radiating elements. The power distribution for the radiating elements was controlled by a cylindrical Electromagnetic Band Gap structured with PIN diodes. Their design allowed the antenna to accept any polarization of signal by controlling the smart antenna through a control system in the radiating elements of the antenna.

Ellinger, Lott, and Bachtold designed an ultra-low powered and low noise amplifier in their research paper entitled Ultra Low GaAs MMIC Low Noise Amplifier for Smart Antenna Combining at $5.2 \mathrm{GHz}$. They were able to design and develop a switchable GaAs MMIC cascode low noise amplifier for smart antennas at $5.2 \mathrm{GHz}$. Their work resulted in a $12.3 \mathrm{~dB}$ gain and a noise figure of $2.4 \mathrm{~dB}$ [25].

In the paper High-performance low-power smart antenna for smart world applications [26], the authors were able to create a smart antenna that consumes low power at $1.5 \mathrm{~mW}$ and is beam-switched. Their work included an improved antenna driver that automates failover and link recovery using a modified Wi-Fi driver. 
In systems where wireless communications need to provide high data transfer rates with high quality signals, smart antennas with beamforming are designed. In the paper Electrically Small and Low-Cost Smart Antenna for Wireless Communication written by Liu, Gao, and Loh [27], they were able to design a compact low-cost low-power smart antenna. They did this by employing the structure of an Electronically Steerable Parasitic Array Radiator (ESPAR) antenna. They called it folded monopole ESPAR antenna because it uses a top disk loaded monopole and folded monopoles which are reduced to less than $1 / 10$ of the wavelength. Their results showed that their antenna can achieve beam scanning in the horizontal plane for over a 360-degree that has a $4.0 \mathrm{dBi}$ gain and a front-back ratio of $20 \mathrm{~dB}$. Beam forming was done by controlling DC voltages applied to the varactors.

In the paper Low-Cost Smart Antennas for Advanced Wireless Systems by Gao and Luo [28], introduces low-cost smart antenna designs where the antennas are electronically steered to its maximum radiation towards the optimal direction of the antenna while nullifying the interfering radiations in the other lobes of the antenna. They were able to discuss smart antennas which were Folded monopole ESPAR, Small director array based smart antenna, Reconfigurable chock ring based smart antennas, and Low-cost smart antenna for satellite communication which is dynamically moving. The researchers provided reviews of those smart antennas that enables low-cost and low-power wireless communication.

\section{THEORETICAL CONSIDERATIONS}

Advanced wireless communications systems require smart antennas. For example, the base stations of cellular sites use omni-directional antennas to enhance its coverage. However, this results to great wastage of power. Smart antennas are commonly used for base stations for its system complexity and high-power consumption. It is ideal to design a low-power smart antenna to remove its drawbacks. To achieve low-power antenna, the radio spectrum must be conserved. It can be done by reducing the interference and increase the frequency spectrum. There are different approaches to smart antenna solutions, Diversity and Adaptive beamforming[29].

The term adaptive antenna, or smart antenna, pertains to a self-phasing system. It re radiates signals from the same direction it was received. Adaptive antennas operate without initial knowledge on which direction it should transmit signal. It is a system consisting of adaptive antenna array and an adaptive processor. Both of the main elements are responsible for the "adaptivity" of the adaptive antenna or smart antenna. [30].

\section{DETAILED PLAN ON HOW TO REVISE OR UPDATE THE SECTIONS OF THE INITIAL MATERIAL}

Once the proper equipment is purchased algorithm testing and development will start. The research will start by reviewing the concepts of the research. Algorithm development will then start based on the learned concepts. After that algorithm validation will then start. This is to ensure that the system really works. With the initial data Written Report writing will start. Focus will be given on the Data and Results and the Validation of Data. Final Algorithm Test and Debugging will then be implemented to determine the accuracy of the system. Before the final publication, there will be a System Review to have a quality publication.

\section{DATA AND RESULTS}

The Antenna Framework was created in this research. Figures $2,3,4$ and 5 shows the antenna patterns created. Initial test was from the paper of [31].

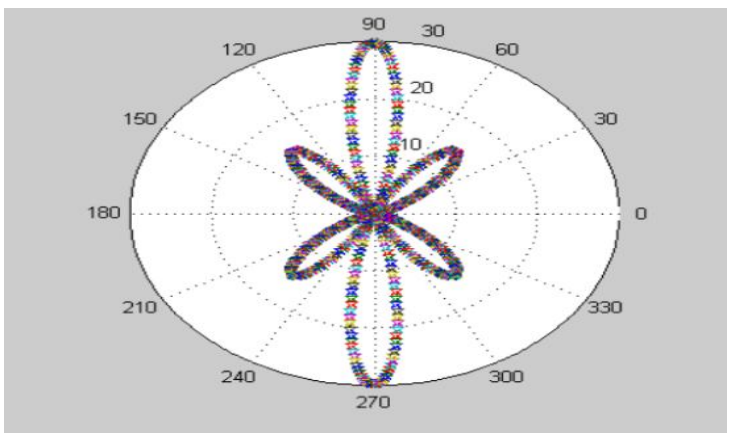

Figure 2: Test 1 Radiation Pattern

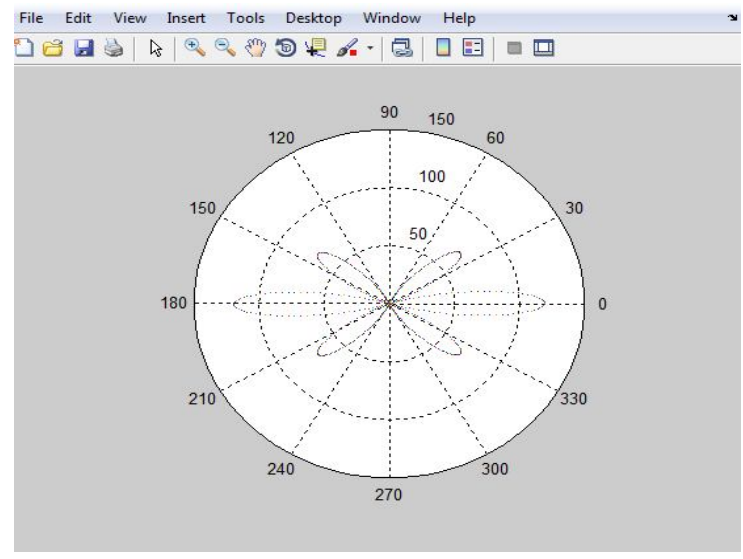

Figure 3: Test 2 Radiation Pattern

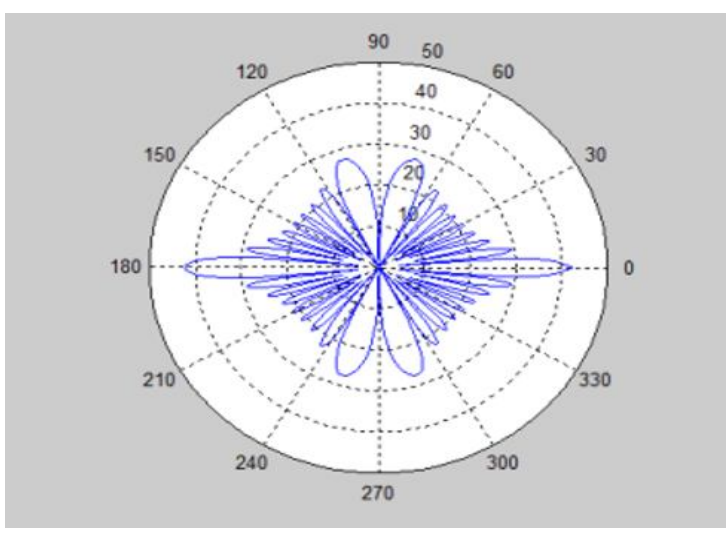

Figure 4: Test 3 Radiation Pattern 


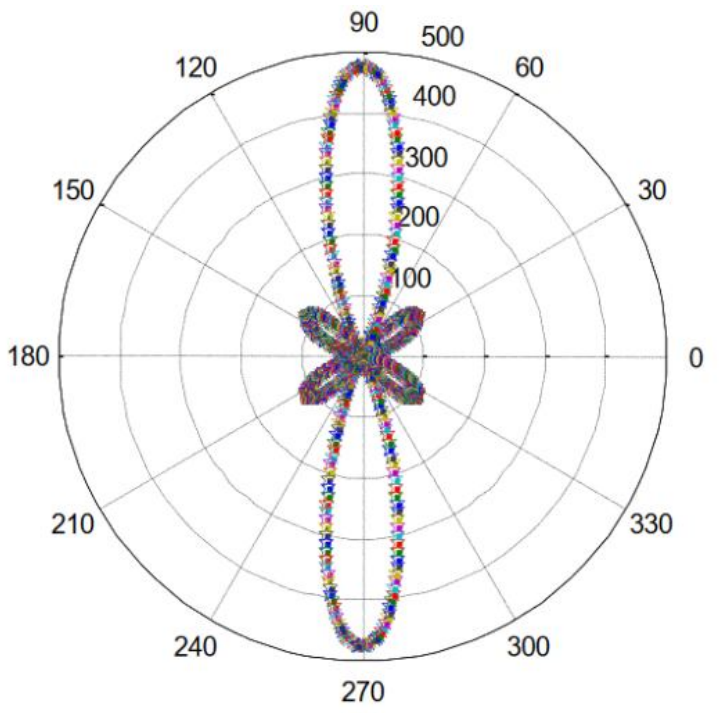

Figure 5: Test 4 Radiation Pattern

The 3D model of the antenna is shown in Figure 6

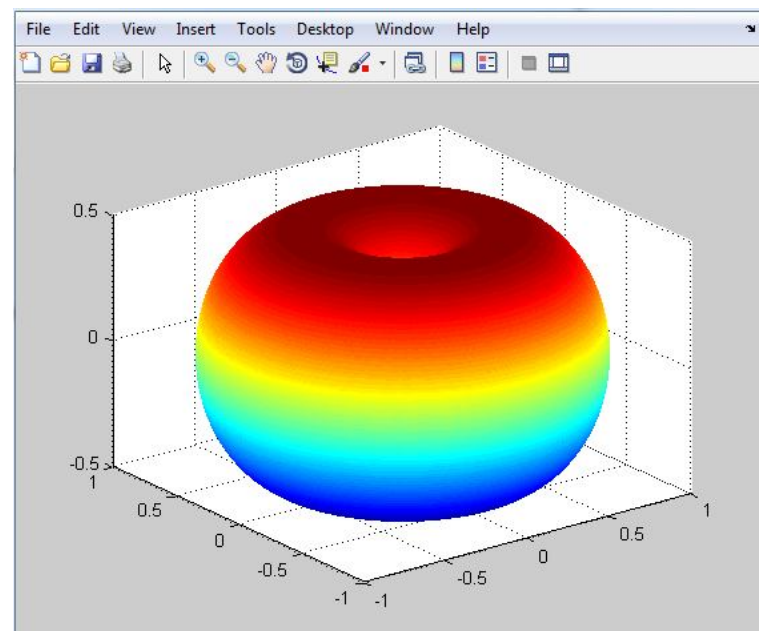

Figure 6: The Smart Antenna created in a 3D Model

\section{CONCLUSION}

Antenna is one of the fundamental concepts of the theory of transmissions of signals. Designing of antennas are one of the concepts that is feasible for signal transmission applications. The design of this low powered smart antenna was implemented using software tools such as MATLAB. Fundamental concepts of the antenna theory were used. The results of this study were considered a success as the results satisfy the condition of the problem.

Antenna design is important in transmission of signal because these are the major components to achieve signal transmission. Antennas are the components that can channel signals from transmitter to receiver.

\section{RECOMMENDATIONS}

The researchers were able to design a model of low powered smart antenna. This design can benefit the users and the providers. It has properties that are feasible and efficient. The researchers were not able to implement the model using hardware tools. The researchers recommended to use other software tools to implement the design. They also recommend implementing the design to other system applications.

\section{REFERENCES}

[1] C. Balanis, "Antenna theory: analysis and design," John Wiley \&Sons. 2016.

[2] W. L. Stutzman and G. A. Thiele, "Antenna theory and design,"John Wiley \& Sons. 2012.

[3] H. Lebret and S. Boyd, "Antenna array pattern synthesis via convex optimization,"Vol. 45, No. 3, pp. 526-532. 1997.

[4] S. A. Schelkunoff, "A mathematical theory of linear arrays," Bell Syst. Tech. J. Vol. 22, No. 1, pp. 80-107,1943.

[5] J. Costantine, Y. Tawk, S. E. Barbin, and C. G. Christodoulou, "Reconfigurable antennas: Design and applications," Proceedings of the IEEE. Vol. 103, No. 3, pp. 424-437, 2015.

[6] R. Christ and R. Wernli, "The ROV Manual (Second Edition)," User Guide for Remotely Operated Vehicles. Pp. 369-385, 2014.

[7] D. Llamocca, and D. Aloi, "Self-reconfigurable implementation for a switched beam smart antenna," Microprocessors and Microsystems. Vol. 60, pp. 1-14. 2018.

[8] Y. Liu and Y. Zhou, "Analysis and estimation of direction of arrival for smart antennas by using a novel filtering algorithm," Procedia Engineering. Vol. 15, pp. 2593-2597, 2011. https://doi.org/10.1016/j.proeng.2011.08.487

[9] L. Hung and S. Wu, "Chapter 2 - Increasing Effective Transmissions Using Smart Antenna Systems," Smart Sensors Networks Communication Technologies and Intelligent Applications. Pp. 19-52, 2017.

[10] N. Kumari, R. Kumar, and R. Bajaj, "Energy Efficient Communication Using Reconfigurable Directional Antenna in MANET," Procedia Computer Science. Vol. 125, pp. 194-200, 2018

[11] D. Schmidt, "U.S. Patent Application No. 09/930,827".

[12] M. Wright, "Using the NRF24L01 $2.4 \mathrm{GHz}$ RF Control Module with the Arduino,"2017.

[13] D. Gray, J. W. Lu, and D. V. Thiel, "Electronically steerableYagi-Uda microstrip patch antenna array," IEEE Transactions on antennas and propagation. Vol. 46, No. 5, pp. 605-608, 1998.

[14] A. Agnihotri, A. Prabhu, and D. Mishra, "Improvement in radiation pattern of Yagi-Uda antenna,"International Journal Of Engineering And Science. Vol. 2, No. 12, pp. 26-35. 2013

[15] K. Kalliola, K. Sulonen, H. Laitinen, O. Kivekas, J. Krogerus, and P. Vainikainen, "Angular power distribution and mean effective gain of mobile antenna in 
different propagation environments,"IEEE transactions on vehicular technology. Vol. 51, No. 5, 823-838. 2002.

[16] A. Salleh, M. Aziz, Z. Zakaria, M. Misran, and N. Hashim, "Electromagnetic radiation effect of action potential based on different antenna position in homogeneous human arm flat and cylindrical shape model using ultra wideband coplanar stripline-fed antenna," International Journal of Emerging Trends in Engineering Research (IJETER). Vol. 8, No. 6, pp. 2557-2562, 2020. https://doi.org/10.30534/ijeter/2020/56862020

[17] C. Courtney and C. Baum, "The coaxial beam-rotating antenna (COBRA): Theory of operation and measured performance," IEEE Transactions on Antennas and Propagation. Vol. 48, No. 2, pp. 299-309, 2000.

[18] S. Shrestha, S. K. Noh, and D. Y. Choi, "Comparative study of antenna designs for RF energy harvesting," International Journal of Antennas and Propagation. 2013.

[19] N. H. Noordin, N. Haridas, A. O. El-Rayis, A. T. Erdogan, and T. Arslan, "Antenna array with wide angle scanning properties," 2012 6th European Conference on Antennas and Propagation (EUCAP). Vol. 6, 2012.

[20] V. V. Mani, and R. Bose, "Smart antenna design for beamforming of UWB signals in Gaussian noise," 2008 International ITG Workshop on Smart Antennas, 2008.

[21] N. Celik, and M. F. Iskander, "Genetic-Algorithm-Based Antenna Array Design for a $60-\mathrm{GHz}$ Hybrid Smart Antenna System," IEEE Antennas and Wireless Propagation Letters. Vol. 7, pp. 795-798, 2008.

[22] A. Singh, J. Kyllonen, S. Caduc, J. Shamblin, M. Garg, and A. Horie, "Compact smart antenna system for improving probability of detection," 2017 IEEE International Symposium on Antennas and Propagation \& USNC/URSI National Radio Science Meeting. 2017.

[23] E. Öström, "Building and experimentally evaluating a smart antenna for low power wireless communication," Mälardalen University: Swedish Institute of Computer Science. 2010.

[24] H. Boutayeb, P. Watson, and T. Kemp, "New reconfigurable power divider based on radial waveguide and cylindrical Electromagnetic Band Gap structure for low power and low cost smart antenna systems," 2014 IEEE Antennas and Propagation Society International Symposium (APSURSI). 2014

[25] F. Ellinger, U. Lott, and W. Bachtold, "Ultra low power GaAs MMIC low noise amplifier for smart antenna combining at 5.2 GHz," 2000 IEEE Radio Frequency Integrated Circuits (RFIC) Symposium Digest of Papers. 2000 .

[26] A. A. Lysko, and M. Mofolo, "High-performance low-power smart antenna for smart world applications," 2014 6th International Congress on Ultra Modern Telecommunications and Control Systems and Workshops (ICUMT). 2014.

[27] H. Liu, S. Gao, and T. Loh, "Electrically Small and Low Cost Smart Antenna for Wireless Communication," IEEE Transactions on Antennas and Propagation. Vol. 60, No. 3, pp. 1540-1549, 2012.

[28] S. Gao, and Q. Luo, "Low-cost smart antennas for advanced wireless systems," 2014 International
Workshop on Antenna Technology: Small Antennas Novel EM Structures and Materials, and Applications (iWAT). 2014.

[29] R. Kawitkar, and D.G. Wakde, "Advances in Smart Antenna System," Journal of Scientific and Industrial Research. Vol. 64, pp. 660-665, 2005

[30] B. Widrow, P. E. Mantey, L.J. Griffiths, and B.B. Goode, "Adaptive Antenna System," Proceeding of IEEE. Vol. 55, No. 12, pp. 2143-2159, 1967. https://doi.org/10.1109/PROC.1967.6092

[31] A. Africa, D. Abaluna, and K, Pimentel, "Monitoring and control systems in agricultural machineries and equipment with a low-power smart antenna system," International Journal of Emerging Trends in Engineering Research (IJETER). Vol. 8, No. 5, pp. 1860-1868, 2020. 